\title{
Forecasting the surface roughness of the workpiece in the round external grinding
}

\author{
Yurij Novoselov ${ }^{1}$, Vladimir Bogutsky ${ }^{1}$, Leonid Shron ${ }^{1, *}$, and Alexander Kharchenko ${ }^{1}$ \\ ${ }^{1}$ Sevastopol State University,33, Universitetskaya St., Sevastopol, 299053, Russia
}

\begin{abstract}
The article gives an analysis of the influence of technological factors on the parameters of the surface roughness during the machining of workpieces with the abrasive tools, based on the use of the modern mathematical apparatus of the theory of probability. It is shown that when processing with abrasive tools, the formation of surface microrelief is directly related to the mechanism of material removal in the area of contact between the workpiece and the tool. The main parameters of the contact zone are its dimensions and parameters of the material removal processes, which are characterized by the probabilities of removal or non-removal of the material. The obtained dependences take into account the parameters of the state of the working surface of the tool and can be used to calculate the basic parameters of the surface roughness in round external grinding. The made estimation of the influence of technological factors on the roughness of the surface of the workpiece being processed allows for the selection of the most significant ones, the changes of which must be taken into account when designing grinding operations.
\end{abstract}

Microroughness of the surfaces of the parts have a significant effect on their performance properties. In this regard, the provision of roughness parameters is provided for the treatment of all surfaces. Criteria for roughness are very diverse. The most frequently used are the arithmetic mean deviation of the $R a$ profile, the largest height of the roughness of the Rmax profile, the height of the unevenness of the profile at ten $R z$ points, and the parameters of the reference surface curve.

When developing a machining operation, the problem arises of predicting the parameters of roughness, which provides a more reasonable choice of tools, cutting modes and other conditions for its implementation. This task is especially important in the design of operations with abrasive tools, which often result in technological processes $[1,2,3]$. The complexity of the problem lies in the fact that the process of interaction between the abrasive tool and the workpiece is stochastic and requires special approaches in the study. Nevertheless, at the present time the system principles of analysis based on the theory-probabilistic approach, system principles of analysis and possessing sufficiently high adequacy of the model, allowing to take into account at designing a large number of technological factors are developed $[3,4,5,6]$. They are the basis for creating techniques, algorithms and programs and can be included in modern CAD systems.

The purpose of the article is to substantiate the method and sequence of solving the problem of predicting surface roughness parameters when machining parts with abrasive tools based on the use of modern mathematical models.
The formation of surface microrelief in abrasive processing is directly related to the patterns of material removal in the area of contact between the workpiece and the tool. The material in the zone is removed by single abrasive grains, i.e. The process of material removal is discrete. However, the number of unit slices is large enough that we can consider it as continuous and use the main provisions of statistical physics. For such a simulation, the concept of the probability of material removal is introduced, consisting in the probability of the event that the material at the point is removed, and the concept of the opposite event is the probability of not deleting the material $[6,7]$.

On the basis of a meaningful description it can be asserted that for the point $M$, Fig. 1, the section of the workpiece that is in the contact zone, the probability of material removal is higher than before the entrance of the section into the zone, and it increases with further passage through the section of the zone. When leaving the zone, the radius-vector of the surface area of the workpiece decreases with circular external grinding by the amount of radial material removal $\Delta r$, a roughness layer $H$ remains on the surface, and their sum is equal to the depth of microcutting $t_{f}$. All three parameters are interrelated and determined under the known cutting conditions and the state of the working surface of the tool. The depth of microcutting is calculated on the basis of the solution of the balance equation of displacement in the technological system (TS), which for the process of round external grinding has the form [7]

\footnotetext{
* Corresponding author: shronlb@,rambler.ru
} 


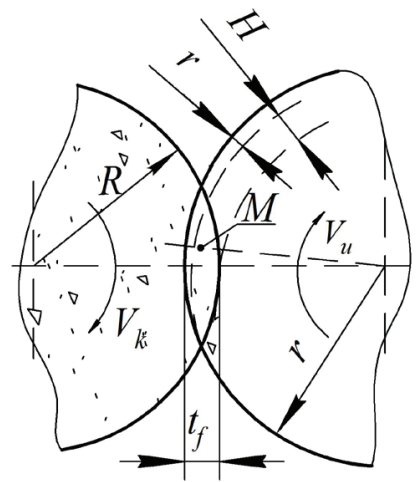

Fig. 1. Process scheme

$$
\Delta A_{s j}=\Delta t_{f j}+\Delta R_{j}+\Delta r_{j-1}+\sum_{k=1}^{n} \Delta y_{y j k}+\sum_{k=1}^{m} \Delta y_{q j k}
$$

where $\Delta A_{s j}, \Delta y_{y j k}, \Delta y_{q j k}$, - respectively, the changes in the center-to-center distance due to the presence of feed, elastic and temperature deformations during the time from the combination of the radius vector of the section of the workpiece surface with the plane passing through the center of rotation of the circle and the workpiece $j-1$ th and $j$-th contacts; $\Delta R_{j}$ - radial wear of the tool; $\Delta r_{j-1}$ radial material removal; $\Delta t_{f j}$ - change of depth of microcutting at $j$-th contact; And - the sum of the increments of the elastic and temperature deformations of the TC elements. A plus sign is assigned if the increment decreases the depth of cut and the minus sign if it increases.

Analysis of equation (1) shows that for forecasting the spatial position of the TS elements it is necessary to have information about the parameters of the quality of the workpiece, the initial profile of the tool, and analytical models determining the relationship of the depth of microcutting, radial material removal, circle wear, cutting forces, elastic and temperature deformations with technological Factors. Such models and examples of solving the balance equation by the iteration method are given in [7].

For the steady-state process of increment of elastic, temperature deformations and $\Delta t_{f j}$ are equal to zero and equation (1) is simplified

$$
\Delta A_{S} j=\Delta R_{j}+\Delta r_{j-1}
$$

For a known value of $\Delta r$, the cutting depth $\Delta t_{f}$ is calculated [7]

$$
t_{f}=0,739 \Delta r+\sqrt{0,546(\Delta r)^{2}+\frac{13,66 V_{u} \Delta r}{K_{S}\left(V_{k} \pm V_{u}\right) n_{g} \sqrt{D_{e} \rho_{g}}}} ;(3)
$$

where $V_{k}, V_{u}$ - the speed of the circle and the speed of the workpiece; $n_{g}$ is the number of abrasive grains per unit of the working surface of the circle; $D_{e}$ - equivalent diameter, $D_{e}=D d /(D+d) ; \rho_{g}$ is the radius of curvature at the top of the grain [12]; $K_{s}$ is the chip formation coefficient, for typical grinding operations is given in $[5$, $8,9]$.
To calculate the roughness parameters, consider the surface before and after the $j$-th contact (Fig. 2).

At a known depth of microcutting, the value of the surface roughness layer is calculated as

$$
H=t_{f}-\Delta r
$$

We determine the position of the midline of the profile after contact, denoting the distance to it from the lower boundary of the roughness layer through $W_{m}$, and the coordinate of the profile point relative to the midline through $y_{m}$.

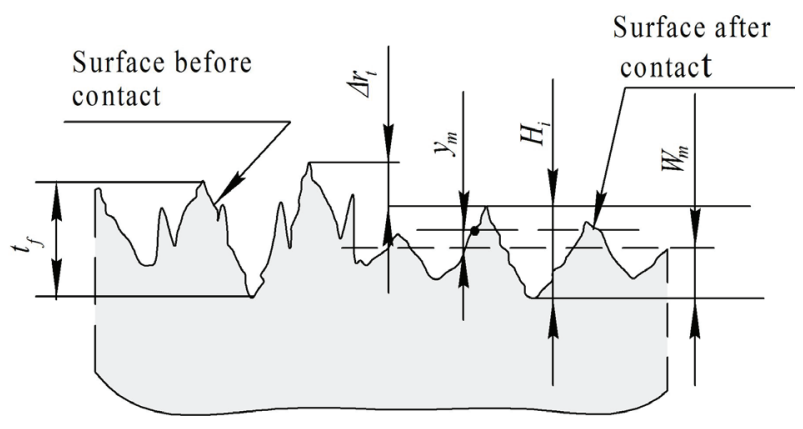

Fig. 2. Surface before and after contact

The average arithmetic deviation of the profile is then calculated

$$
R_{a}=\frac{1}{L} \int_{0}^{L}\left|y_{m}\right| d x
$$

where $L$ is the length of the base portion of the surface microrelief.

The integral in the dependence (5) determines the sum of the areas of the protrusions and valleys. They can also be determined by integration with respect to the variable $y_{m}$.

Since for the area of the protrusions $d F_{l}=t_{p} L d y_{m}$ and the area of the depressions $d F_{2}=\left(1-t_{p}\right) L d y_{m}$, Ldym, then

$$
\begin{aligned}
& R_{a}=\int_{0}^{\infty} t_{p} d y_{m}+\int_{-\infty}^{O}\left(1-t_{p}\right) d y_{m} \\
& \text { or } R_{a}=\int_{0}^{\infty}(1-P(M)) d y_{m}+\int_{-\infty}^{0} P(M) d y_{m},
\end{aligned}
$$

where $t_{p}$ is the relative length of the reference profile, the mathematical expectation of which is equal to the probability of not removing the material; $P(M)$ is the probability of material removal, calculated by [7].

The dependence for calculating $R a$ is greatly simplified if we use the position $[10,11]$ that the random process describing the ordinates of the profile is stationary and normal. For such a process, the mathematical expectation $R a$ is proportional to the mean square deviation $\sigma Y_{m}$ of the random ordinates $Y_{m}$ of the profile points 


$$
M\left[R_{a}\right]=\sqrt{\frac{2}{\pi} k(0)}=\sigma_{Y m} \sqrt{\frac{2}{\pi}},
$$

where $k(0)$ is the value of the correlation function, for the interval $x_{1}=x_{2}$ is equal to the variance of the random variable $Y_{m}$.

To determine the root-mean-square deviation $\sigma_{Y m}$, we calculate the function $F_{Y m}\left(y_{m}\right)$ and the probability density $f_{Y m}\left(y_{m}\right)$ of the distances $Y_{m}$. For positive and negative values of $y_{m}$.

$$
\begin{aligned}
F_{Y m}\left(y_{m}\right) & =P\left(Y_{m}<y_{m}\right)=\lim _{L \rightarrow \infty} \frac{L-\sum b_{m i}}{L}=P(M) \\
& f_{Y m}\left(y_{m}\right)=v G \sum_{i=0}^{n}\left(W_{m}+y_{m}-i \Delta r\right)^{v-1} \times \\
& \times\left\{\exp \left[-G \sum_{i=0}^{n}\left(W_{m}+y_{m}-i \Delta r\right)^{v}\right]\right\}
\end{aligned}
$$

where $n$ is determined from the condition [8]

$$
\begin{gathered}
W_{m}+y_{m}-n \Delta r \geq 0 ; \quad W_{m}+y_{m}-(n+1) \Delta r \leq 0 ; \\
G_{k}=\frac{\sqrt{\pi D_{e}} \Gamma(m+1) \Gamma(\chi) \chi K_{s} C_{b}\left(V_{k} \pm V_{u}\right) n_{g}}{\Gamma(m+\chi+3 / 2) V_{u} H_{u}^{\chi}} \\
v=m+\chi+1 / 2
\end{gathered}
$$

where $m$ and $C_{b}$ are the parameters of the shape of the cutting edge of the abrasive grain [12]; $\chi$ - grain distribution parameter in the depth of the working surface of the tool; $H_{u}$ - the depth of the working layer of the tool, within which the number of abrasive grains is calculated; $\quad \Gamma(m+1) ; \quad \Gamma(\chi) ; \quad \Gamma(m+\chi+3 / 2)$ are the corresponding gamma functions.

Comparison of the probability densities of the ordinates of the profile, calculated from equation (9) and Gaussian law, confirmed the hypothesis that the random process - the ordinates of the polished profile surface - is close to normal. For such a process, $y_{m}=0$ и $P(M)=0,5$,

$f_{Y m}\left(y_{m}\right)=\frac{1}{\sigma_{Y m} \sqrt{2 \pi}}$.

On the basis of this, the second part of equation (9), separated by curly braces, is 0.5 , and the average arithmetic deviation of the profile, taking into account equation (8), is defined as

$$
R_{a}=\frac{2}{\pi G v \sum_{i=0}^{n}\left(W_{m}-i \Delta r\right)^{v-1}}
$$

The distance $W_{m}$ from the deepest depression to the midline of the profile is calculated from the condition

$$
y_{m}=0, P(M)=0,5, G \sum_{i=0}^{n}\left(W_{m}-i \Delta r\right)^{v-1}-\ln 2=0 .
$$
$W_{m}$

At the value of the radial removal of the metal $\Delta r \geq$

$$
W_{m}=\left(\frac{\ln 2}{G}\right)^{1 / v} \text {. }
$$

As shown in [7], with the value of the radial metal removal $\Delta r \geq W_{m}-W_{m} \approx 0,5\left(t_{f}-\Delta r\right)$ and at particular values $m=0,5$ (the shape parameter of the cutting edge of the abrasive grain [12] and $\chi=1,5$ (the grain distribution parameter for the depth of the working surface of the tool), $R a$ can be calculated as:

$$
R_{a}=\frac{\sqrt{2} V_{u} H_{u}^{3 / 2}}{\pi^{2} K_{S}\left(V_{k} \pm V_{u}\right) n_{g} \sqrt{D_{e} \rho_{g}} \sum_{i=0}^{n}\left(W_{m}-i \Delta r\right)^{3 / 2}}
$$

$$
\begin{aligned}
& R_{a}=\frac{\sqrt{2} V_{u} H_{u}^{3 / 2}}{K_{S}^{0.4}\left(V_{k} \pm V_{u}\right)^{0.4} n_{g}^{0.4} D_{e}^{0.2} \rho_{g}^{0.2}} \\
& \Delta r \geq W_{m} ; \text {. }
\end{aligned}
$$

where $H_{u}$ is the depth of the working layer of the tool, within which the number of abrasive grains is calculated.

Equation (12), in its structure and magnitude of the exponents for variable factors, is close to the power relations in the literature, but, unlike them, reflects the physical nature of the process of shaping and corresponds to the basic principles of the theory of dimensions.

The maximum height of the roughness of the Rmax profile and the height of the profile irregularities along the ten $R z$ points can be calculated from the layer value in which the surface roughness is distributed and the mathematical expectation of the distances $Y_{1}, Y_{2} \ldots Y_{5}$ from the upper boundary of the layer to the five higher profile points and distances $W_{1}, W_{2} \ldots W_{5}$ from the lower boundary of the layer to the five lowest points of the profile (Fig. 3).

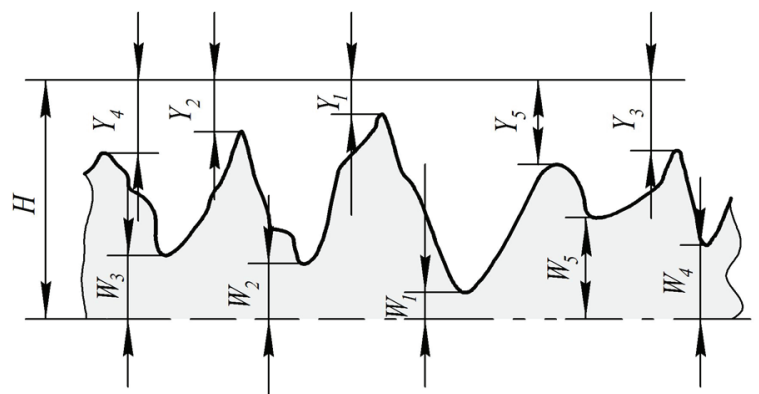

Fig. 3. Scheme for calculating the maximum height of the profile irregularities and the height of the irregularities by ten points 
The distances considered are random and independent, on the basis of this [7, 13]

$$
\begin{gathered}
M\left[R_{\text {max }}\right]=H-M\left[Y_{1}\right]-M\left[W_{1}\right] ; \\
M\left[R_{z}\right]=H-\frac{1}{5} \sum_{i=1}^{5} M\left[Y_{1}\right]-\frac{1}{5} \sum_{i=1}^{5} M\left[W_{1}\right] ; \\
D\left[R_{\max }\right]=D\left[Y_{1}\right]-D\left[W_{1}\right] ; \\
D\left[R_{z}\right]=\frac{1}{25} \sum_{i=1}^{5} D\left[Y_{1}\right]-\frac{1}{25} \sum_{i=1}^{5} D\left[W_{1}\right] .
\end{gathered}
$$

For a stationary process close to normal, we can assume that the distances from the upper boundary of the roughness layer to the most protruding vertices of the profile are distributed according to laws analogous to the distribution of distances from the depressions to the lower boundary of the layer. From this follows the approximate equality of mathematical expectations and variances $Y_{j}$ and $W_{j}$..

If the density distribution of grain vertices along the depth of the tool is modeled by a power law, then the number of vertices of the grains passing beyond the last contact of the surface area with the circle is determined from equation [7]

$$
\lambda=\frac{\widetilde{A}(\chi) \chi\left(V_{k} \pm V_{u}\right) n_{g} \sqrt{\pi D_{e}}}{\widetilde{A}(\chi+3 / 2) V_{u} H_{u}^{\chi}} u+1 / 2=Q u \chi+1 / 2
$$

To calculate the mathematical expectations and variances, the corresponding distribution functions $F_{W}(w)$ are defined. Each of the deepest depressions of the microrelief can be viewed as a trace from a separate peak of the abrasive grain, so the probability of events $P\left(W_{1}<w\right), P\left(W_{2}<w\right), \ldots P\left(W_{5}<w\right)$, which consists in the fact that the distance from the lower boundary to the first, second, ..., fifth cavities is less than some fixed values of $\mathrm{w}$, is determined by the probability of passing through the layer of section from $w=0$ to $W$ by at least one, two, ..., five vertices of grains . The probability of passing through a section of a section of length $L$ of at least one vertex is determined by the consequence of the Poisson law

$$
F_{W 1}(w)=P\left(W_{1}<w\right)=1-e^{-\lambda L} .
$$

The probability of passing at least two vertices is less than the probability of passing at least one vertex by the probability of passing only one tops

$$
F_{W 2}(w)=P\left(W_{2}<w\right)=P\left(W_{1}<w\right)-\frac{\lambda L}{1 !} e^{-\lambda L} .
$$

The probability of passing through a cross section of at least three, four, and five grains

$$
F_{W 3}(w)=P\left(W_{3}<w\right)=P\left(W_{2}<w\right)-\frac{\lambda^{2} L^{2}}{2 !} e^{-\lambda L}
$$

$$
\begin{aligned}
& F_{W 4}(w)=P\left(W_{4}<w\right)=P\left(W_{3}<w\right)-\frac{\lambda^{3} L^{3}}{3 !} e^{-\lambda L} \\
& F_{W 5}(w)=P\left(W_{5}<w\right)=P\left(W_{4}<w\right)-\frac{\lambda^{4} L^{4}}{4 !} e^{-\lambda L}
\end{aligned}
$$

After a consistent determination of the distribution functions, probability densities, mathematical expectations, and dispersion of the distances $W_{i}$ and $Y_{i}$ [7], the equations for calculating $M[R \max ], D[R \max ]$, $M[R z], D[R z]$

$$
\begin{gathered}
M\left[R_{\text {max }}\right]=H-2 \Gamma\left(\frac{2 \chi+3}{2 \chi+1}\right)\left(\frac{Q}{L}\right)^{\frac{2}{2 \chi+1}} \\
D\left[R_{\max }\right]=2\left[\Gamma\left(\frac{2 \chi+5}{2 \chi+1}\right)-\Gamma^{2}\left(\frac{2 \chi+3}{2 \chi+1}\right)\right]\left(\frac{Q}{L}\right)^{\frac{4}{2 \chi+1}} ; \\
M\left[R_{z}\right]=H-\frac{2}{5}\left(\frac{Q}{L}\right)^{\frac{2}{2 \chi+1}} \sum_{i=1}^{5} \frac{1}{(i-1) !} \Gamma\left(\frac{2 i \chi+i+2}{2 \chi+1}\right) ; \\
D\left[R_{z}\right]=\frac{2}{25}\left(\frac{Q}{L}\right)^{2 \chi+1} \sum_{i=1}^{5} \frac{1}{(i-1) !}\left[\Gamma\left(\frac{2 i \chi+i+4}{2 \chi+1}\right)-\right. \\
\left.-\frac{1}{(i-1) !} \Gamma^{2}\left(\frac{2 i \chi+i+2}{2 \chi+1}\right)\right]
\end{gathered}
$$

where $Q$ is denoted by $Q=\frac{\Gamma(\chi+1,5) V_{u} H_{u}^{\chi}}{\Gamma(\chi) \chi\left(V_{k}+V_{u}\right) n_{g} \sqrt{\pi D_{e}}}$.

The values of the parameter $\chi$ in calculating the probability of material removal, mathematical expectations and variance $R \max$ and $R z$ can be different, since different parts of the real curve of the probability density of grain distribution over the depth of the tool $f(u)$ are modeled. For a particular value $\chi=1,5$, equations (13) and (15) take the form

$$
\begin{gathered}
M\left[R_{\text {max }}\right]=H-2 \sqrt{\frac{2 V_{u} t_{f}^{3 / 2}}{3 n_{g}\left(V_{k} \pm V_{u}\right) L \sqrt{D_{e}}}} ; \\
M\left[R_{z}\right]=H-2,95 \sqrt{\frac{V_{u} t_{f}^{3 / 2}}{n_{g}\left(V_{k} \pm V_{u}\right) L \sqrt{D_{e}}}} .
\end{gathered}
$$

An example of an analytical calculation of the surface roughness parameters for the process of circular external grinding of samples of steel 9X2 with a diameter of $50 \mathrm{~mm}$ in circles $1300 \times 40 \times 12724 \mathrm{~A}$ F60 L $4 \mathrm{~V}$ is given in Table 1.

In the calculations, it is assumed: $K_{s}=0,9 ; V_{k}=35 \mathrm{~m} / \mathrm{s}$; $V_{u}=0,25 \mathrm{~m} / \mathrm{s}$; Transverse feedrate $\Delta A_{s}=3,33 \cdot 10^{-9} \mathrm{~m}$ per one turn of the workpiece; $n_{g}=5.2 \cdot 10^{6} 1 / \mathrm{m}^{2} ; \rho_{g}=21 \cdot 10^{-6}$ $\mathrm{m}$, the value of the base length $L=0.8 \cdot 10^{-6} \mathrm{~m}, \Delta R=0.01 r$. 
Table 1. Example of calculating the height parameters of the surface roughness

\begin{tabular}{|c|c|c|}
\hline $\begin{array}{c}\text { № in } \\
\text { order }\end{array}$ & Parameter & The equation \\
\hline 1 & $\Delta r=3,3 \cdot 10-6 \mathrm{~m} / \mathrm{turn}$ & $(2)$ \\
\hline 2 & $t_{f}=11,5 \cdot 10^{-6} \mathrm{~m}$ & $(3)$ \\
\hline 3 & $H=8,2 \cdot 10^{-6} \mathrm{~m}$ & $(4)$ \\
\hline 4 & $R a=1,0 \cdot 10^{-6} \mathrm{~m}$ & $(11)$ \\
\hline 5 & $R m a x=7,3 \cdot 10^{-6} \mathrm{~m}$ & $(17)$ \\
\hline 6 & $R z=6,5 \cdot 10^{-6} \mathrm{~m}$ & $(18)$ \\
\hline
\end{tabular}

A comparison of the calculated and experimental values of the relative reference length of the profile for the grinding conditions considered in the example is given in [7].

Table 2 compares the calculated and experimental data obtained with oscillating grinding of blanks with longitudinal feed $S_{x}$ from steel 9X2 with a diameter of $50 \mathrm{~mm}$ in circles of $1300 \times 40 \times 12724 \mathrm{~A} \mathrm{~F} 60 \mathrm{~L} \mathrm{4V}$, HRC $55 \ldots 60$. The repetition of experiments at each point of the experimental design is taken equal to nine

For the most part of the processing modes, the deviation of the calculated data from the experimental data does not exceed $20 \%$, which makes it possible to recommend the considered sequence of predicting surface roughness parameters when designing grinding operations. The greatest deviation of the calculated data from the experimental ones is observed for mode 2 . It is assumed in the calculations that the number of abrasive grains on the working surface does not depend on the regime while it is known that with decreasing speed and increasing feed, the probability of grain breaking and tearing out of the bundle increases Leads to a reduction in the number of cutting edges on the working surface of the tool.

Table 2. Experimental and calculated values of surface roughness during grinding with changing cutting conditions

\begin{tabular}{|c|c|c|c|c|c|c|}
\hline \multirow{2}{*}{$№$} & \multirow{2}{*}{$\begin{array}{c}V_{k}, \\
\mathrm{~m} / \mathrm{s}\end{array}$} & \multirow{2}{*}{$\begin{array}{c}V_{u}, \\
\mathrm{~m} / \mathrm{s}\end{array}$} & $\begin{array}{c}S_{x}, \\
\mathrm{~mm} / \mathrm{s}\end{array}$ & $\begin{array}{c}S_{t}, \\
\mathrm{~m} / \mathrm{s}\end{array}$ & \multicolumn{2}{|c|}{$R a, \mu \mathrm{m}$} \\
\hline 1 & 35 & 0,66 & 16,6 & 0,533 & 1,17 & 1,2 \\
\hline 2 & 20 & 0,33 & 16,6 & 0,533 & 1,57 & 3,0 \\
\hline 3 & 20 & 0,66 & 8,3 & 0,533 & 1,54 & 1,6 \\
\hline 4 & 20 & 0,66 & 16,6 & 0,266 & 1,08 & 1,2 \\
\hline 5 & 35 & 0,33 & 8,3 & 0,533 & 1,20 & 1,0 \\
\hline 6 & 35 & 0,33 & 16,6 & 0,266 & 0,85 & 0,6 \\
\hline 7 & 35 & 0,66 & 8,3 & 0,266 & 0,83 & 0,7 \\
\hline 8 & 20 & 0,33 & 8,3 & 0,266 & 1,10 & 0,8 \\
\hline 9 & 27 & 0,50 & 12,5 & 0,400 & 1,10 & 1,0 \\
\hline 10 & 27 & 0,50 & 12,5 & 0,400 & 1,10 & 0,9 \\
\hline
\end{tabular}

Thus, it is recommended that the calculation of the surface roughness parameters during grinding be performed in the following sequence:

- at the first stage, the input variable operations are defined: the parameters of the state of the working surface of the tool, its geometric characteristics, the parameters of the workpiece, the grinding mode;

- in the second stage, the solution of the displacement balance equation, equations (1) and (2), calculate the radial material removal, the depth of micro cutting and the thickness of the surface roughness layer.

- in the third stage, select the dependence for calculating the roughness parameters from the dimensional analysis data and update the initial data if necessary;

- in the fourth stage, the required parameters for the surface roughness are calculated from the equations given; Give an assessment of their reliability;

If the parameters do not match, given by the designer, the selection of the cutting mode and the characteristics of the abrasive tool are corrected.

If necessary, the calculations are repeated with new values of the input variables.

\section{References}

1. S.M. Bratan, Quality assurance and improving processing stability during the finishing and fine grinding, Cutting \& tool in technological system. International Scientific-Technical Collection. Edition 68, P. 34-39 (2005)

2. Stephen Malkin, Changsheng Guo, GRINDING TECHNOLOGY. Theory and Applications of Machining with Abrasives, (Industrial press, New York,. - P.372. 2008)

3. Yu.K. Novoselov, D.A. Kainov, S.M. Bratan, The construction of the model of a ground surface on the basis of a stochastic description of the properties of the tool, Nauk. pr. Donec. nac. tehn. un-tu. Ser.: Mashinoobladnannja, Issue 7. P.17-31 (2010)

4. Ioan D Marinescu, [et al.], Tribology of abrasive machining processes, (Published in the USA by Brent Beckley, William Andrew, Inc. Cover Art P.764. 2004)

5. S.N. Korchak, Productivity of process of grinding of steel parts (M.: Mashinostroenie, P. 280 1974)

6. A.V. Korolev, Yu.K. Novoselov, Theoretical and probabilistic fundamentals of abrasive processing. (Part 1. State of the working surface of the abrasive tool, Saratov: Izdatel'stvo Saratovskogo universiteta, P.158. 1987)

7. Yu.K. Novoselov, Dynamics of surface shaping in abrasive processing, (LAP LAMBERT Academic Publishing. Saarbrucken, Deutschland, P.317 2017)

8. A.N. Reznikov, Abrasive and diamond processing of materials: Handbook (M.: Mashinostroenie, P.391, 1977) 
9. Robert I. King, Robert S. Hahn, Handbook of modern grinding technology (Publ. Chapman and Hall, New York, P.369. 1986)

10. A.P.Husu, Yu.R.Vitenberg, V.A. Palkov, Roughness of surfaces: the theoretical-probabilistic approach., (M.: Nauka, P.344. 1975)

11. Yu.R. Vitenberg, Application of the correlation theory for the evaluation of polished surface, Vestnik mashinostroenija, Issue 1, P. 55-57 (1969)

12. L.V. Khudobin, Analysis of the geometry of abrasive grains, Mechanical engineering, electro instrumentation. Saratov: Privolzh. Book Publishing house. P. 6-20. (1966)

13. A.D. Wentzel, Course of the theory of random processes (M .: Nauka. Fizmatlit, P.420, 1996) 